\title{
Disrupting Complacent Systems
}

\author{
Laura Grindstaff and Linda F. Bisson
}

\begin{abstract}
In this chapter, we characterize the academy as a "complacent system" resistant but not impervious to change, and we discuss how to overcome that resistance in the pursuit of diversity, equity, and inclusion. We outline best practices for disruption drawing upon what we learned during the implementation of the NSF ADVANCE program on our campus. We then offer some thoughts on the steps ahead, including resources for sustainability.
\end{abstract}

Keywords Institutional transformation · Inclusive diversity $\cdot$ Peer-to-peer learning $\cdot$ Sustainability $\cdot$ Community consensus $\cdot$ Myth of meritocracy $\cdot$ Building resilience

\section{Introduction}

If existing social systems generally work well for most people in a group, organization, or community, these systems can become complacent and resistant to change. As we considered the long road ahead in sustaining the initiatives launched under the UC Davis ADVANCE-IT grant, we reflected collectively on what we had learned, what had proved most useful in moving toward a more-inclusive campus culture, and what advice we wished we had at the onset. We have documented our insights in this book as a way to catalyze deeper thinking about barriers to inclusion, optimal ways of addressing them, and how to tackle challenges that remain. We share our insights and experiences hoping they will benefit those in other institutions, both in and outside the academy, as they undertake their own diversity, equity, and inclusion efforts in pursuit of a new normal.

\footnotetext{
L. Grindstaff $(\varangle)$

Department of Sociology, University of California, Davis, Davis, CA 95616, USA

e-mail: lagrindstaff@ucdavis.edu
}

\section{F. Bisson}

Department of Viticulture and Enology, University of California, Davis, Davis, CA 95616, USA

e-mail: lfbisson@ucdavis.edu 


\section{Inclusive Diversity as a Social Good}

As Stewart and Valian (2018) observe, for some people, the notion of fairness by itself is reason enough to support diversity, on the presumption that "excellence" more or less exists equally across all social groups and members of under-represented groups simply need access, a seat at the table. Provided all groups have more or less equitable access to the resources and opportunities needed to develop academic excellence, this is a reasonable assumption. But even if this proviso were true, we concur with Stewart and Valian (2018: 41) that "diversity is not only fair but smart, because diversity [can] promote excellence" (our emphasis). When diversity is inclusive-when everyone is a fully valued participant_-diversity among faculty facilitates innovation, broadens areas of study, and improves outcomes for students because it communicates the message that there is room for everybody, even "people like me."

Without inclusion, diversity may be little more than what Marvasti and McKinney (2011) call "enlightened assimilation." Assimilation "allows" members of historically under-represented groups to join a common perspective already established. Being inclusive, by contrast, means welcoming ideas and perspectives that might differ from the status quo and/or differ from your own. Often what results from respectful discussion is a synthesis which is stronger than any individual perspective. In and outside of academia, inclusive environments that encourage people to share divergent perspectives can improve critical thinking and problem-solving skills as well as develop greater self-confidence (Chang et al., 2006). Diversity per se does not necessarily lead to improved outcomes if that diversity is not inclusive (Jayne \& Dipboye, 2004; Stewart \& Valian, 2018). Inclusion, rather than assimilation, leads to the benefits most often associated with diversity_including academic excellence.

That the faculty of research-intensive universities in the U.S. are less diverse than they should be is well-documented, as is the persistence of bias against women faculty and faculty of color (see Stewart \& Valian, 2018). Barriers to inclusive diversity are complex. Higher education is guided by the principles of meritocracy, and although white women and under-represented minority (URM) scholars are not denied advancement under these principles, they may face greater scrutiny and work harder for recognition; as well, their contributions may be undervalued and colleagues may not readily see them as idea-generators, innovators, or discoverers (see Conway, 2018). All faculty like to think of themselves as fair-minded and objective, especially in their interactions with, and evaluations of, colleagues. But not all thought and action is fully conscious, and objectivity is an ideal that can only ever be approximated because there no way to push culture aside and exist outside of itthere is no way to eliminate one's own subjectivity. Nor is this always a desirable goal. Recognizing the role subjectivity can play in decision-making, and, depending on the nature of the decision, reducing its impact, strengthens the meritocracy for everyone. The fact that much thought is outside of conscious awareness further complicates things, of course. Implicit social cognition, a normal and inevitable aspect of brain functioning, can and does generate implicit bias; yet because it can't be seen and we 
believe ourselves be in control of our thoughts and actions, we think implicit bias doesn't apply to, or affect, us. This is a real challenge in pursuing inclusive diversity as a social good.

\section{Disrupting Complacent Systems: Lessons from UC Davis ADVANCE}

Let's assume you're convinced that diversity, equity, and inclusion (DEI) are desirable and you know that your institution could do better. Assuming the resources are there, how do you make the case for change and how do you implement that change? Below we share "best practices" for disrupting complacent systems, drawing on our own insights and experiences institutionalizing the five interlocking ADVANCE initiatives on our own campus.

\subsection{Make the Case for Change}

In making the case for change, people have to understand what you're talking about. Explain concepts simply and unambiguously; clearly describe questions and issues. What does "inclusive" really mean? How can it be measured? Why is the current culture not inclusive? How will becoming more inclusive change the current culture? Why is this change important? Avoid disciplinary jargon as best you can, as it compromises effective communication.

Understand that some concepts are inherently difficult to grasp-implicit bias, for example-and be prepared to work at it, incrementally if necessary. Whereas explicit or conscious bias is well-known, implicit bias is often interpreted incorrectly as forgotten explicit bias, leading people to adamantly deny that they have it. Change is unlikely if people don't understand the nature of the problem and deny it exits (more on implicit bias in the next section). Other concepts_-"social justice," for example-may be too vague to be helpful, as they have different and sometimes conflicting meanings depending on the context of use, even within academia. People can interpret this term as meaning anything from a socialist redistribution of wealth to acknowledging the legacy of a historical wrong to apologizing for offending someone perceived as easily offended. This "definition drift" can hinder consistent communication about, and comprehension of, the nature of the problem to be addressed.

Even when barriers to inclusion are generally recognized, majority-group members may still doubt the existence of a specific barrier or how "real" it is. Is there really a barrier or does the aggrieved individual simply choose not to persevere? If 
she finds an environment to be hostile or toxic, is it hostile or is she just hypersensitive? Here, documenting where, when, and how systems generate or sustain bias is important to making the invisible visible. Data will help all community members to see the barriers that only some may face (more on this later).

\subsection{Conversation, Not Confrontation}

The most critical lesson we learned is the importance of community-wide understanding of the nature of bias as an essential first step. (Again, in our case, "community wide" refers primarily to the STEM fields on our campus). Conversation works better than confrontation in facilitating collective learning. Some conversations will be uncomfortable, even difficult. It is important that people are open to hearing each other because if things get confrontational, listening ends. For example, if someone expresses an unpopular opinion, rather than challenging them or telling them they are wrong, ask why they think that way. Keep the conversation going. Views change during conversation whereas they solidify under confrontation. Making abstract concepts accessible is also challenging if they provoke a defensive response. Referring to unconscious bias as "unconscious racism," without adequate explanation, feels threatening and can generate denial and confrontation. An alternative approach is to first explain the differences between conscious and unconscious learning, or explicit versus implicit cognition. This can work well for STEM audiences. Start with a discussion of "preference" rather than "bias." If asked what their favorite color is, most people will have a ready answer. Articulating why that is their favorite color is more difficult because the motivation may be implicit.

Cognitively, implicit preference is an orientation toward the routine, the established, or the expected. Preferences are generally associated with positive emotion, even a vague feeling that "all is right with the world." Our expectations that the people around us will act in ways we consider to be "normal" for their gender or racial category form a positive cognitive feedback loop. Thus, bias may be rooted in a preference for the familiar, as the familiar is tied to the generation of positive emotion (Duhigg, 2012). These expectations may limit opportunity or access for non-majority groups, although there is no malicious intent. In contrast, the term "bias" is generally associated with negative emotions, such as fear, anger, or hatred. When we began our work, people often told us they did not believe they had implicit bias, because they did not hate or wish ill for any group. We also often heard that if some individuals did possess implicit bias, it could not be easily changed, if at all. Logically, though, if an apparent bias is really an unconscious preference for the familiar, the familiar can be changed.

If terms like "bias" or "unconscious racism" generate defensiveness and derail meaningful exchange, consider using more neutral terms such as "preference" in initial conversations with colleagues, especially when they more accurately describe the situation. Unconscious or implicit preference is an easier concept to hear/receive and helps pave the way for understanding the nature of implicit bias. Meeting people 
where they are requires patience (particularly if where they are is far from where you want them to be) but if sustainable institutional transformation is the end goal, it's a strategic step along the way.

\subsection{Develop Trust and Build Consensus}

Creating a more inclusive work climate cannot be imposed from the outside; it has to reflect the will of the whole community. Yet it's challenging to make the case for change when not everyone experiences the same kind or degree of bias. If the policies and practices of an organization or institution work well for the majority, how do you ensure the organization works for the collective, not just the majority? How do you get everyone on board?

As discussed in detail in the Chapter, 'Barriers to Inclusion: Social Roots and Current Concerns,' perspective is linked to social location in that social location yields a specific line of sight. A critical step in disrupting complacent systems is enabling majority-group members to value the perspectives, the sightlines, of others-and further, to trust and defer to those perspectives on issues of bias. Privilege is often invisible to those who possess it and highly visible to those who do not. In our fence analogy, people who easily see over the fence have a different perspective on the value of fences than the people whose sightlines are obscured, and the views of those who experience the fence as a barrier must be respected/trusted. Here again, learning about implicit bias along with other barriers to inclusion-identity exclusion, lack of belonging, stereotyping, microaggressions, work-life imbalance, etc.-was key. Individuals with the clearest view of the problem (not the clearest view of how well the status quo functions) must play a lead role in defining the change needed.

At the same time, building a strong consensus for change is not the exclusive work of a marginalized minority within a community. A marginalized group can define the change needed but alone cannot make it happen. Indeed, no one group, let alone a single individual or handful of individuals, has enough power or possesses the breadth of perspective required to make the case for change and develop a roadmap for getting there. Rather, the will to enact change must come from everyone; the bias experienced by the few needs to become the problem of the many. Consequently, any institutional transformation process must have at its core a focus on vetting. Initiative leaders need to listen to all voices and perspectives, including those of sceptics and naysayers.

Part of listening is making people feel that their views are important and are being heard. Naysayers - those who deny there is a problem to be fixed-have things to teach you, and they, too, are part of the community. Including all perspectives in discussions of barriers to inclusion does not diminish the greater barriers that some face compared to others. In our experience, such inclusion builds greater empathy. By listening, we learned, somewhat counter-intuitively, to avoid the term "ally." Although developing a cadre of white male allies was recommended by colleagues from other grant-receiving institutions, we found that conceptualizing 
white men as "allies" stigmatized them as "others" - others who may "get it," but as "others" nonetheless. In a truly inclusive community, there are no others. We developed the practice of referring to majority-group members committed to inclusion as stakeholders-to reinforce their vested interest in a common goal.

Thus, any transformation process should get the entire community talking. An anonymous quote gathered during the final external evaluation of our grant put it best: "Having the ADVANCE grant got the campus talking about things that we hadn't really talked about before. It became an exponentially growing conversation, and now we're constantly talking about these issues and advancing faculty diversity. It has raised the cognition across the entire campus." Although all five ADVANCE initiatives contributed to these "exponentially growing conversations," no initiative did this better than the STEAD training in implicit bias, discussed in the chapter titled "Assessing Institutionalized Bias." A key reason we were able to raise awareness, is that we engaged a critical mass of faculty. Across the five initiatives and the internal and external advisory boards, nearly 50 faculty joined in our efforts on an ongoing basis. All initiatives met multiple times per year and produced tangible outcomes that reinforced our collective commitment to transformation. To seek inclusivity, the ADVANCE team itself had to model inclusivity.

\subsection{Prioritize Peer-To-Peer Learning}

Passive learning by being lectured at by experts might work well for certain purposes but we found that active learning through self-exploration and peer-to-peer dialogue was more effective. Peer-to-peer learning facilitates a faculty context rooted in mutual respect, shared values, and common experiences.

Of course, peer-learning can be developed using expert sources. Individuals committed to developing expertise about an issue can self-identify and form a learning group; this group can then dispense what they have learned to others. Our STEAD (Strength Through Equity and Diversity) training, which focused on faculty recruitment and hiring processes, employed this method. We benefited from the fact that many other institutions had already developed programs for identifying implicit bias in academic hiring - a key resources here was the STRIDE training used at the University of Michigan (STRIDE stands for Strategies and Tactics for Recruiting to Improve Diversity and Excellence). We adopted and adapted elements of these various programs to our own organizational needs, tailoring them to the institutional culture of our campus. Faculty volunteers read from the available literature and consulted with experts to learn about implicit bias and subsequently developed an interactive workshop on how to recognize and address implicit bias in the faculty recruitment process. We then required that all members of faculty search committees participate in the training, every three years. Other faculty and administrators took advantage of the training as well. Once a critical mass had been trained, their knowledge spread to other faculty members in their departments and programs via discussions of search outcomes. Productive discussions of strategies for creating a 
more-inclusive academic community grew naturally from this collectively-produced knowledge.

Meanwhile, the ADVANCE leadership team and the members of the Policy and Practices Review Initiative engaged in extensive learning of their own. They reviewed the literature on implicit bias and invited to campus speakers with expert knowledge of implicit bias and other barriers to inclusion to lead general discussions with the team. They involved the appropriate faculty committees that regularly engage these issues: the Committee on Academic Personnel, the Committee on Affirmative Action and Diversity, and the Committee on Faculty Welfare, in addition to the oversight Executive Council of the Davis Division of the Academic Senate. They held annual retreats for the entire ADVANCE team during which we sought the wisdom of the external advisory committee as well as representatives from the other nine campuses of the University of California system. It was through these multiple and overlapping conversations that we were able to put flesh on the bones of the five core initiatives that comprised our ADVANCE program. These initiatives were the vehicles for establishing a "new normal" for diversity, equity, and inclusion on the campus-an ongoing effort.

\subsection{Know that Leadership and Resources Matter}

One factor that all individuals recognize about institutional transformation is the challenge of the process itself-and the need for committed leadership and a strong base of support working together with common purpose. The commitment of the top levels of administration needs to be visible and real, especially when prioritizing resources. If commitment to change is top-down only, however, the process will not work. It is all too easy in academia to chase resources by ensuring that requests for local resources match the stated goals of the central administrators holding the purse strings. As such, merely allocating resources may create a transient commitment in those academic units receiving them. As we've repeatedly emphasized, leadership for sustainable change must come from the community itself. For this reason, we engaged many faculty members in the vetting of ADVANCE initiatives. We held open seminars, panel discussions, and workshops; we reported out to the community on a regular basis; we created a website that would attract those interested in learning more; we sought the advice and engagement of the Academic Senate; we were fully transparent and welcomed advice and comments from all institutional stakeholders. Faculty talked and we listened, and listened again.

This is not to suggest that resources are trivial. Most certainly they are not. In fact, generous funding made possible our successes by underwriting all of our hard work; in turn, the successes born of our hard work helped to justify the generous funding. The resources supporting our efforts far beyond the dollar amount of the NSF ADVANCE-IT grant itself (\$3.7 million). The UC Davis Chancellor at the time, Linda Katehi, was also the grant's Principle Investigator. Written into the grant proposal was a promise from the campus to hire 16 new STEM faculty (representing roughly 
$\$ 5$ million annually) and provide ongoing support for a number of the proposed ADVANCE initiatives, including the Center for the Advancement of Multicultural Perspectives in Science (CAMPOS). The campus made good on its promises. The 16 new faculty were hired as "CAMPOS Scholars," with access to mentors, networking and professional development opportunities, and award programs. Most important, CAMPOS Scholars became part of an interdisciplinary, multicultural "think tank" enabling faculty to work together collaboratively on projects with the potential for significant community impact. CAMPOS is a jewel of the UC Davis ADVANCE program — and the price tag for its development was high.

\subsection{Obtain Your Own Data, Know Your Own Data}

Academics, across the many disciplines, are generally data-driven. They require a strong rationale for the need to change. This, in turn, requires not merely quoting the observations of others but also collecting, analyzing, and fully knowing one's own institutional data. When we were initially making the case for mandatory implicit bias training for faculty-search committees, the most common objection voiced by faculty was that no existing data actually showed the university had a problem with bias at the point of hire, as low numbers of women and/or people of color within any given field or discipline does not prove bias per se if the pool of available $\mathrm{PhDs}$ is also low. Of course, there were no data clearly showing the opposite, either, but we took the point.

The most recent data on our campus addressed salary equity and advancement for faculty after the point of hire, and found no significant differences among groups at the same academic rank within departments, although there were large differences in salary structures between disciplines, even within the same college-for example, Sociology has a lower salary scale than Economics. Yet equity in salary and advancement once faculty are already at the university are not the only or necessarily the best indicators of inclusive diversity. It became important, therefore, to document baseline or starting data in order to show progress or programmatic effectiveness. In our case, trend lines for greater diversity continued, although some argued, not unreasonably, that the increases we saw under the ADVANCE grant might have happened without the grant. Ultimately, our goal was to create an environment in which noninclusion was acknowledged as a problem for all STEM faculty in the institution, so that our institutional transformation efforts would be widely supported. And we did accomplish that goal.

We also learned the shortcomings of our own data as we tried to compare centrallyheld data to those of local units. We found that, although hiring data matched across the two levels fairly well, retention data did not: the numbers aligned, but the qualitative data revealed discrepancies between the actual reasons faculty gave for leaving the campus (or even academia entirely) at the local level and the reasons officially recorded in the central database. Locally, climate did appear to sometimes play a role in their decisions. 
Thinking comprehensively about which data are needed to measure what, clarifying how data should be collected and managed, and identifying the key metrics for analyzing data-all these elements are important in evaluating the impact of inclusion programs. At very large and complex institutions, it is a daunting task. Yet as inclusive diversity becomes a higher priority in the academy, it will become increasingly important to gather data with inclusive diversity in mind from the outset, and not a task to be cobbled together after-the-fact.

\subsection{Get at the "Why" and Not Just the "What" of Bias}

Once the case for change is made, you need a strategic plan with concrete action steps to improve diversity, equity, and inclusion. This, too, is a difficult process, even with strong community consensus. A major challenge is that not everyone within the community will need the same support structures and programs to experience a more-inclusive cultural climate. We found that faculty from different areas within STEM had very different experiences with respect to bias in group work, assignment of credit, lab setup, the availability of course buy-outs and other resources, and access to mentoring networks, among other measures. This variability, caused by local disciplinary cultures, has to be accounted for in developing a roadmap for institutional transformation and seeking ways to enact it that will have the most positive impact.

One consistent barrier that cut across different constituencies was lack of work-life imbalance, especially for women faculty. The academic culture that currently prevails arose in the era of single-career couples, and much of what is "familiar" is still based on the gendered realities of two individuals (typically a man and woman) enabling the career of one (typically the man). In the Chapter, 'Barriers to Inclusion: Social Roots and Current Concerns,' we describe this as the "ideal worker norm." Factors such as overwork (the 60- hour faculty work week) and dedication to work (putting career ahead of family) still inform our ideas about how to achieve success in STEM. This creates barriers for dual-career couples, whose increasingly widespread presence in academia has not automatically unsettled this traditional view of the "ideal worker" (here, the "ideal scientist"). Although a shift is underway toward valuing the impact of "work across time" versus "time spent on work," it has been slow in coming. Relatedly, there is a shift away from a STEM culture of work-life imbalance to one of work-life integration in some pockets of academia and occasionally in the corporate world, but this societal change, too, is slow and its advancement uneven. UC Davis has relatively generous work-life integration policies for faculty (see the Chapter, 'Work-Life Integration in Academia: From Myth to Reality'), but more can and should be done, particularly with regard to securing affordable childcare for faculty at the lowest salary scales. 
Other common barriers reported by faculty pertained to the devaluation of teaching, service, advising, and community-engagement, all seen as largely incompatible with research excellence in STEM according to prevailing notions of meritocracy. To better reward what we collectively value in the academy, the ADVANCE team advocated for changes to the criteria for outstanding achievement by adding to, but not replacing, the existing criteria. Such efforts had already been underway on campus - including the institution of the "Step Plus" system of advancement for faculty, and the addition of a diversity statement to candidate portfolios for faculty undergoing review (see the Chapter, 'Assessing Institutionalized Bias').

\subsection{Question the Myth of Meritocracy}

When they appear, barriers to inclusion in the academy are often sustained by the myth of an objective, value-free meritocracy. Indeed, this is perhaps the toughest nut to crack. But it must be addressed if equity and excellence both are to be achieved. STEM fields often demonstrate a "monoculture of cultural perspective," meaning that the values and views of dominant groups become the values and views of the larger academic enterprise itself. When we created CAMPOS, we often heard from faculty that only one perspective prevailed in science-the scientific method, and dedication to this method-and culture had nothing to do with it.

Rather than point out that the scientific method is itself a product of culture, with objectivity as its core value, and rather than point out the many historical examples (and even some current ones) of sexist and racist scientific experimentation, consider instead asking faculty about the ways in which culture might impact the contexts of research that actually produce scientific findings. These questions might include:

- which issues/problems do scientists decide to study?

- what motivates them to study these issues?

- which questions do they pose, and why?

- what norms, values, or principles prevail in their labs, if any?

- who works in their labs?

- who do faculty collaborate with, and why?

- who-which communities-does their research serve?

- do research opportunities reflect the priorities of external funding agencies?

- If so, what are those priorities, and why?

- are scientific journals equally hospitable to all types of findings-for example, null findings as well as those that confirm a theory or hypothesis?

Talking about the contexts of research, the conditions both material and intellectual in which research is practiced, can serve to underscore the fact there is no way to "bracket off" culture and exist outside of it. This is, in our view, a good thing, because culture, while often incalcitrant, in not impervious to change. 


\subsection{Build the Capacity for Resilience}

One of the key findings from our Social Sciences Research Initiative was the importance of building resilience in the face of barriers to inclusion. By resilience we don't mean an individualistic, "pull-up-your-bootstraps" capacity to bounce back from hardship and persist regardless of circumstance. In the academy, building resilience takes multiple forms, including: (1) providing adequate support, mentoring, and sponsorship opportunities; (2) learning about barriers to inclusion, because knowledge of those barriers and their social origins helps to mitigate their power; and (3) understanding the connection between resilience and self-worth. We think of selfworth as being shaped by internal and external currencies. Internally, it is the sense of having and meeting a personal value system that affirms a positive self-concept. Externally, self-worth can be enhanced if it is confirmed by others; alternatively, it can be undermined by others' negative views - especially if those others play an evaluative role and are generally perceived to be fair-minded and objective. For faculty who experience marginalization, implicit bias training can minimize the damaging effects of negative evaluations by highlighting the potential role of unconscious bias rather than or in addition to presumed impartial assessments of merit. Thus, such training is important not just for those who might want to check their own biases but also for those who might suffer from biases left unchecked.

In thinking about the first currency of self-worth-having an internal value system and the desire to live up to those values-we are reminded once again of the ideal worker norm and the conflict it creates, especially for women, between succeeding at work and succeeding at home. If a woman's core value system prioritizes the latter, she might reject a career option that jeopardizes it. In many Latinx cultures rooted in Catholicism, placing family first is a strongly-shared (and widely internalized) cultural value. The value is embraced by both men and women, but is typically realized in traditional, gender-specific ways (men provide for the family, women care for it). For some Latinas, then, rejecting a particular career path-e.g., a familyunfriendly one in STEM-may reflect the need to retain self-worth as well as adhere to community norms.

As we proceeded with the ADVANCE-IT project, we developed several programs aimed at reinforcing resilience-including the LAUNCH committees that served to link new faculty to a network of valuable resources and wise mentors on the campus, and various CAMPOS events and programs that supported the center's community of scholars committed to diversity, equity, and inclusion.

\section{Sustain Change}

We are under no illusions that sustainable institutional transformation on our campus will be smooth from this point forward. In addition to the matter of necessary resources, there is the matter of "culture," which is more powerful than people tend to 
give it credit for. In the academy, the views and values of majority-group members, consciously and unconsciously held, tend to represent the academic culture that prevails for all, especially with regard to meritocracy; this works fine for some, even most, but creates barriers to full inclusivity. Creating a genuine culture of "we" can, in fact, be done (Dovidio, et al., 2009; Gaertner et al., 1993). Shared principles, including those that define merit, can be upheld in an inclusive way-not to dilute excellence, but in fact to reinforce it.

We offer the following suggestions for sustaining a culture of inclusion. First, it is important to continue the discussion of barriers to inclusion. Learning must be sustained over time. A one-time workshop is unlikely to have lasting impact unless its lessons and insights are put to regular use. Devising ways to engage in continuous conversation is important. This can be accomplished through regular and periodic events, interactive blogs or other on-line forums, and informal but enabled gatherings such as book clubs, film clubs, and speakers' series-provided there is no pandemic preventing congregation. It is also important for women and URM faculty to have access to separate as well as integrated spaces for peer learning, as separate spaces typically feel safer for sharing and validating personal experiences. The more positive the environments for learning, such as over a meal or at a pleasant location, the deeper the commitment to learning may be.

Second, alongside and in tandem with sustained learning through conversation, institutionalized programs that help keep an inclusive climate alive are critical to fund and maintain - on our campus these would include CAMPOS, the Work-Life Integration Program, the Committee on Affirmative Action and Diversity, the Committee on Faculty Welfare, the Feminist Research Institute, the Office of Diversity, Equity, and Inclusion (which oversees the LAUNCH mentoring committees), and Academic Affairs (which oversees sexual harassment training, the STEAD training, and the Capitol Resource Network). The UC Davis ADVANCE program developed a number of these lasting programs through the five initiatives that organized our DEI efforts.

Third, frequent community self-assessment is essential. We suggest that a team representing the diversity of the community — and, ideally, including representatives from the sorts of programs listed above- - be created and meet regularly to address any issues that arise. This would help ensure that different campus units are not duplicating efforts, talking past each other, or dropping the ball on a problem. The values of diversity and inclusion have to be continually and visibly reinforced until they become the new normal.

\section{National and International Resources}

Of course, the NSF ADVANCE-IT program is not the only source of support of diversity, equity, and inclusion in higher education, nor is the U.S. alone if providing resources. The National Science Foundation offers a variety of grant opportunities 
under the ADVANCE umbrella which target STEM. ${ }^{1}$ In the spirit of peer-to-peer learning, there now exists the ADVANCE Resource and Coordinating Networka program that connects ADVANCE-funded programs with each other, enabling participants to interact. ${ }^{2}$ The group holds annual meetings to exchange information and best practices across a host of institutions committed to addressing systemic bias.

Other organizations offer more formalized engagement. The Athena SWAN (Scientific Women's Academic Network) originated in Great Britain, with the goal of fostering gender equality in STEM fields. Although initially focused on STEM, the program is now under the auspices of "ADVANCE HE" and covers non-STEM fields as well ${ }^{3}$ (the "H-E" stand for higher education). Member institutions engage in self-assessment and commit to uphold a charter of common principles, ${ }^{4}$ with periodic review to ensure continued commitment. The Athena SWAN program has spread internationally. In Australia, it is housed under the SAGE program (Science in Australia Gender Equity). ${ }^{5}$

The American Association for the Advancement of Science has developed a similar program, SEA Change, with SEA standing for STEMM Equity Achievement (the extra "M" broadens the acronym to include medicine). ${ }^{6}$ The SEA Change program focuses on addressing systemic, structural inequalities, of which gender is a component. Like the Athena SWAN program, SEA Change institutions commit to a core group of principles of inclusion and formally apply for membership. ${ }^{7}$ Membership is based upon review of a detailed self-assessment; each institution is granted a status based on the self-assessment. SEA Change uses a rating system with status increasing from Bronze to Silver or Gold depending upon the nature and degree of progress towards inclusion. ${ }^{8}$ Initially, members receive bronze status and periodic reassessment is mandatory for continued membership as well as advancement to a higher level. These types of organizations serve to formalize the commitment to change and require continued progress and evaluation. They also provide member institutions with resources and opportunities for peer learning.

DEI efforts also extend beyond the academy. The CEO pledge for Action aims to advance diversity, equity, and inclusion across the workforce. ${ }^{9}$ Whether this is more than window dressing remains to be seen. As noted in the Chapter, 'From Affirmative Action to Inclusion,' diversity programs owe their origins and initial growth primarily to the business world - they developed not out of a desire for fairness necessarily,

\footnotetext{
${ }^{1}$ Description of the National Science Foundation ADVANCE program https://www.nsf.gov/fun ding/pgm_summ.jsp?pims_id=5383.

${ }^{2}$ ADVANCE Resource and Coordinating Network https://www.equityinstem.org/.

${ }^{3}$ ADVANCE HE program https://www.ecu.ac.uk/equality-charters/athena-swan/.

${ }^{4}$ Charter of Athena SWAN core principles https://www.ecu.ac.uk/equality-charters/athena-swan/ about-athena-swan/.

${ }^{5}$ SAGE program https://www.sciencegenderequity.org.au/category/athena-swan/.

${ }^{6}$ STEMM Equity Achievement Change program https://seachange.aaas.org/.

${ }^{7}$ SEA Change core principles https://seachange.aaas.org/about/principles.

${ }^{8}$ SEA Change Levels https://seachange.aaas.org/about/pillars.

${ }^{9}$ CEO pledge https://www.ceoaction.com/pledge/ceo-pledge/.
} 
but to avoid litigation. Corporations in the 1970s and 80s created diversity trainings in order to avoid civil rights lawsuits charging gender and/or racial discrimination. As with faculty and administrators in academia, today's CEOs agree to a core set of principles and commit their organizations to change. The network provides guidelines for change and enables interaction among members on best practices.

What many institutions of higher education have that corporations may not is deep belief in a set of academic ideals, what Stewart and Valian (2018) call "academic virtues," that can be harnessed to facilitate sustained change: the search for truth, the freedom to pursue all ideas, respect for knowledge, and a belief in merit. These are fundamental virtues that can produce research excellence, provided we define and operationalize them with diversity and inclusion in mind.

\section{Conclusion}

In and outside of the academy, DEI programs make visible a commitment to institutional transformation, yet their effectiveness is by no means guaranteed. Since complacent systems are most comfortable remaining complacent, ongoing "disruption" is necessary. Otherwise, complacent systems drift back to complacency and the commitment to change gives way to the ease of staying the same. To avoid this, the necessity of change must be continuously documented; the consensus to act secured through dialogue and debate; a plan of action devised and accepted by all stakeholders; and considerable resources-financial and intellectual-devoted to implementing and sustaining the plan. The role of resources cannot be overstated. Ideally, institutions of higher education should think of external grant support such as that provided by the NSF ADVANCE programs as "seed" funding to shift the needle on the dial; cultivating broad and sustained institutional change will take much more.

Regarding this last point, it seems to us that faculty colleagues with deep expertise in racial and gender inequality are an under-valued and untapped resource. Yes, STEM faculty have to lead the charge if STEM fields are the ones targeted for change. But these fields don't exist in isolation from the rest of the university, just as science doesn't exist in isolation from the broader society. Consider the COVID-19 pandemic, which, at the time of the writing, is seemingly winding down. The pandemic has disproportionately affected black and brown Americans along with those shouldering child- and elder-care responsibilities, predominantly women. Clearly both biological and cultural expertise are relevant to understanding and addressing the causes and impacts of such a crisis.

Moreover, cultural expertise must extend beyond the psychology of individuals. Stereotyping, implicit bias, microaggressions - all are important to understand and challenge. But they aren't the whole of the story, because even people who are consciously anti-racist in attitude can enable structural racism-by deciding where to live, choosing where to school their kids, or, closer to home, by allocating the fewest resources to the most diverse pockets of an organization or institution. This last point references a sobering reality: in the University of California system, diversity 
is inversely related to funding (Newfield, 2016, 2020). This pattern holds across universities, across campuses within the system, and across disciplines on a campus, including our own. Indeed, we note the considerable irony of devoting so much time and money to diversify STEM at UC Davis while already-diverse social science and humanities departments struggle with insufficient resources to meet student demand. That DEI initiatives here and elsewhere have blossomed in this context underscores both their limitations and their unrealized potential to mobilize more collaborative and wide-ranging transformation.

It would be naïve to conclude that any single program or initiative will solve the problem of systemic bias in the academy, let alone end structural racism, sexism, heterosexism, or any other "ism." Even with concerted effort and tremendous resources, our own campus is far from perfect on this score. Another lesson we learned, then, is that you have to keep trying. Keep talking, keep listening, and keep searching for new ways to understand and address the problems. Nothing happens without collective, sustained commitment. Given the interconnectedness of different disciplines within a university, of different universities within higher education, and of higher education within the society at large, disrupting one complacent system makes trouble, however small, for them all.

\section{References}

Conway, L. (2018). The disappeared: Beyond winning and losing. Computer, 51(10), 66-73. https:// doi.org/10.1109/MC.2018.3971344

Chang, M. J., Denson, N., Sáenz, V., \& Misa, K. (2006). The educational benefits of sustaining cross-racial interaction among undergraduates. The Journal of Higher Education, 77(3), 430-455. https://doi.org/10.1080/00221546.2006.11778933

Dovidio, J. F., Gaertner, S. L., \& Saguy, T. M. (2009). Commonality and the complexity of "we": Social attitudes and social change. Personality and Social Psychology Review., 13, 3-20.

Duhigg, C. (2012). The power of habit. Random House.

Gaertner, S. L., Dovidio, J. F., Anastasio, P. A., Bachman, B. A., \& Rust, M. C. (1993). The common in group identity model: Recategorization and the reduction of intergroup bias. European Review of Social Psychology, 4, 1-26.https://doi.org/10.1080/14792779343000004

Jayne, M. E. A., \& Dipboye, R. L. (2004). (2004) Leveraging diversity to improve business performance: Research findings and recommendations for organizations. Human Resource Management, Winter, 43(4), 409-424.

Marvasti, A. B., \& McKinney, K. D. (2011). Does diversity mean assimilation? Critical Sociology, 37(5), 631-650. https://doi.org/10.1177/0896920510380071

Newfield, C. (2016). The great mistake: How we wrecked public universities and how we can fix them. John Hopkins University Press.

Newfield, C. (2020). When are access and inclusion also racist? Remaking the University, posted June 28, 2020. http://utotherescue.blogspot.com/2020/06/when-are-access-and-inclusion-also. $\mathrm{html}$

Stewart, A., \& Valian, V. (2018). An inclusive academy: Achieving diversity and excellence. Massachusetts Institute of Technology. 
Open Access This chapter is licensed under the terms of the Creative Commons Attribution 4.0 International License (http://creativecommons.org/licenses/by/4.0/), which permits use, sharing, adaptation, distribution and reproduction in any medium or format, as long as you give appropriate credit to the original author(s) and the source, provide a link to the Creative Commons license and indicate if changes were made.

The images or other third party material in this chapter are included in the chapter's Creative Commons license, unless indicated otherwise in a credit line to the material. If material is not included in the chapter's Creative Commons license and your intended use is not permitted by statutory regulation or exceeds the permitted use, you will need to obtain permission directly from the copyright holder.

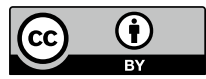

